EXTENDED REPORT

\title{
Dactylitis in psoriatic arthritis: a marker for disease severity?
}

\author{
J E Brockbank, M Stein, C T Schentag, D D Gladman
}

See end of article for authors' affiliations

.....................

Correspondence to:

Dr D D Gladman, Centre

for Prognosis Studies in the Rheumatic Diseases,

Toronto Western Hospital,

399 Bathurst St ECW 5-

034B, Toronto, Ontario

M5T 2S8, Canada; dafna.

gladman@utoronto.ca

Published OnLine First

22 July 2004

Accepted 20 June 2004

\begin{abstract}
Aim: To describe dactylitis in a large cohort of patients with psoriatic arthritis followed prospectively in a specialist clinic, and identify whether it is associated with a worse prognosis.

Methods: Between 1979 and 1999, 537 patients were registered in the psoriatic arthritis clinic and entered onto a longitudinal database. Patients were followed prospectively at six to 12 month intervals according to a standard protocol, and all information was entered onto a database. The database was searched for patients with dactylitis. Descriptive statistics were used to describe the population and $\chi^{2}$ tests to relate dactylitis to radiographic changes.

Results: Dactylitis was documented in 260 patients (48\%); 69\% of the episodes were recorded at presentation to the clinic. Dactylitis affected feet only in $65 \%$ of cases, hands only in $24 \%$, and both hands and feet in $12 \%$. Recurrent dactylitis occurred in $44 \%$ of the patients. Increased radiological progression was noted in digits showing dactylitis compared with those without dactylitis ( $50 \%$ v 38\%, respectively; $p<0.0001)$.

Conclusions: Dactylitis is common among patients with psoriatic arthritis. It most offen affects the feet, in an asymmetrical distribution. It is associated with a greater degree of radiological damage than occurs in digits not affected by dactylitis.
\end{abstract}

soriatic arthritis is an inflammatory arthritis associated with psoriasis. ${ }^{1}$ Although there are no widely accepted criteria for the diagnosis or classification of this type of arthritis, the diagnosis is made easier in a patient with the typical features of the disease including involvement of distal interphalangeal joints, asymmetrical distribution, the presence of spondyloarthropathy, or the presence of inflammation of the whole digit (dactylitis). ${ }^{2}$

Dactylitis, or the "sausage digit", has long been recognised as one of the cardinal features of psoriatic arthritis, ${ }^{3}$ occurring in up to $40 \%$ of patients. ${ }^{4}$ It is often found in the other spondyloarthropathies, especially the reactive arthropathies. It can be seen in gout, sarcoidosis, flexor sheath infections, and sickle cell disease, but very rarely in rheumatoid arthritis. ${ }^{56}$ Only recently, with the arrival of ultrasound and magnetic resonance imaging (MRI), have we begun to gain insight into the underlying pathology of dactylitis. ${ }^{78}$ These new methods of investigation have shown that dactylitis is primarily the result of inflammation of the digital flexor tendon sheaths. An MRI study revealed very few cases with true synovitis of the small joints of the digit (one of 36 joints examined). ${ }^{8}$ Ultrasound imaging in another population, however, showed joint involvement in 52\% of digits, ${ }^{7}$ compared with none in the Italian cohort. The prevalence and natural history of dactylitis among patients with psoriatic arthritis is poorly described. Radiographs carried out at time of dactylitis have revealed periostitis and joint space narrowing, but it is not known whether dactylitis itself is associated with erosive joint damage and deformity or is a comparatively benign condition.

Our aims in this investigation were to identify the frequency of dactylitis among patients with psoriatic arthritis and to investigate whether it is associated with more severe radiological changes.

\section{METHODS}

\section{Patients}

All data in this study were derived from the cohort of patients attending the psoriatic arthritis clinic at the University of
Toronto. Patients are referred from primary, secondary, and tertiary care and may be de novo cases or patients with established disease. They are registered in the clinic if they have an inflammatory arthritis associated with psoriasis and if other rheumatological conditions have been ruled out. ${ }^{9}$ These patients are followed prospectively and assessed according to a set protocol at six to 12 month intervals by physicians trained within the department in the assessment of psoriatic arthritis. At each clinic visit a complete history is elicited and physical examination and laboratory assessments are done according to a standard protocol. Radiological examinations of the peripheral joints and the spine are carried out at one to two year intervals. All information collected is entered onto an ORACLE database.

The clinical assessment of actively inflamed joints and the evaluation of damaged joints, both clinically and radiologically, have proved reliable in our clinic. ${ }^{10}{ }^{11}$ Between 1978 and 1999, 537 patients were registered in the psoriatic arthritis clinic and entered onto the longitudinal database. The database was searched for the presence of dactylitis, and all records were reviewed to confirm the presence of dactylitis and the sites at which it occurred.

\section{Definition of dactylitis}

Dactylitis—defined as a diffuse swelling of a digit-may be acute, with painful inflammatory changes, or chronic, where the digit remains swollen despite the disappearance of acute inflammatory changes. The presence of acute painful inflammatory dactylitis is recorded for each digit; chronic clinically non-inflammatory (non-tender) dactylitis is recorded separately. In this study only acute inflammatory dactylitis was considered.

\section{Radiology}

Radiographs of the hands, feet, and spine including the sacroiliac joints are taken at two year intervals or earlier if indicated. They are reviewed by a single observer and scored according to the modified Steinbrocker technique, as previously validated in this population. ${ }^{11}$ In this scoring 
Table 1 Disease characteristics of psoriatic arthritis patients with dactylitis $(n=260)$

\begin{tabular}{|c|c|c|}
\hline Variable & Presentation & $\begin{array}{l}\text { First visit with } \\
\text { dactylitis }\end{array}$ \\
\hline \multicolumn{3}{|l|}{ Age at onset (years) } \\
\hline Psoriasis & $28(13.1)$ & - \\
\hline Psoriatic arthritis & $34(11.6)$ & - \\
\hline \multicolumn{3}{|l|}{ Disease duration (years) } \\
\hline Psoriasis & $13(11.2)$ & 14 (12.0) \\
\hline Psoriatic arthritis & $7(7.9)$ & $8(8.6)$ \\
\hline Age (years) & $41(12.3)$ & $42(12.8)$ \\
\hline Male sex & $57 \%$ & \\
\hline \multicolumn{3}{|l|}{ Arthritis pattern } \\
\hline 1. Distal & $8 \%$ & $7 \%$ \\
\hline 2. Oligoarthritis & $14 \%$ & $13 \%$ \\
\hline 3. Polyarthritis & $44 \%$ & $43 \%$ \\
\hline 4. Back only & $0.4 \%$ & - \\
\hline 5. Back+1 & $4 \%$ & $4 \%$ \\
\hline 6. Back+2 & $6 \%$ & $5 \%$ \\
\hline 7. Back+3 & $23 \%$ & $28 \%$ \\
\hline Number of active joints & $11(9.1)$ & $11(9.2)$ \\
\hline Number of clinically deformed & & \\
\hline $\begin{array}{l}\text { joints } \\
\text { Dactylitis (\% present) }\end{array}$ & $\begin{array}{l}2(5.3) \\
33.5 \%\end{array}$ & $3(6.1)$ \\
\hline Psoriasis (\% present) & $95 \%$ & $97 \%$ \\
\hline Nail lesions (\% present) & $84 \%$ & $85 \%$ \\
\hline \multicolumn{3}{|l|}{ Drug treatment } \\
\hline None & $14 \%$ & $11 \%$ \\
\hline NSAID & $47 \%$ & $42 \%$ \\
\hline Gold, chloroquine, sulfasalzine & $5 \%$ & $6 \%$ \\
\hline Imuran, methotrexate & $13 \%$ & $15 \%$ \\
\hline PUVA, retinoid & $6 \%$ & $9 \%$ \\
\hline Corticosteroid & $15 \%$ & $18 \%$ \\
\hline
\end{tabular}

Values are mean (SD) or per cent.

NSAID, non-steroidal anti-inflammatory drug; PUVA, psoralen plus ultraviolet $A$ irradiation.

system each joint is rated as follows: 0 , normal; 1 , soft tissue swelling or juxta-articular osteopenia; 2, erosions present; 3 , erosions and loss of joint space; 4, complete destruction. All information is entered into an electronic database for analysis. Radiographic progression of disease was determined by comparing pre- and post-dactylitis radiographs. The predactylitis radiograph was the one taken closest to the onset of dactylitis or, where that was not available (such as at the first visit), the one taken at the time of clinical presentation. The post-dactylitis radiograph was the most recent one taken. Progression of radiological damage was considered to have occurred if the score for any of the finger joints in a given digit (metacarpophalangeal, proximal interphalangeal, and distal interphalangeal joints for digits 2 to 5 in the hands, and metacarpophalangeal and interphalangeal joint of the thumbs) had increased between $x$ rays to at least a score of 2. Owing to the difficulties in interpreting $x$ rays of the small joints of the feet, only the metatarsophalangeal joints and first interphalangeal joint were scored.

\section{Statistical analysis}

Descriptive statistics were used to describe the patient population. The absence or presence of dactylitis in individual digits among the patients with dactylitis was related to the absence or presence of radiological progression for that digit; $\chi^{2}$ tests were used to assess the association between dactylitis and radiological progression.

\section{RESULTS}

\section{Prevalence}

Of the 537 patients registered in the psoriatic arthritis clinic between 1979 and 1999, 260 (48\%) had at least one episode of acute dactylitis. Only the first episode is considered in this report. One hundred and eighty patients $(69 \%)$ had dactylitis at their first clinic visit. Thus the prevalence of dactylitis at
Table 2 Distribution of dactylitis among digits $(n=259)$

\begin{tabular}{lllll}
\hline Digit & R hand & L hand & R foot & L foot \\
\hline 1 & $12(4.6)$ & $6(2.3)$ & $39(15.1)$ & $38(14.7)$ \\
2 & $30(11.6)$ & $27(10.4)$ & $47(18.2)$ & $38(14.7)$ \\
3 & $25(9.6)$ & $15(5.8)$ & $41(15.8)$ & $29(11.2)$ \\
4 & $8(3.1)$ & $11(4.2)$ & $73(28.2)$ & $58(22.4)$ \\
5 & $6(2.3)$ & $7(2.7)$ & $36(13.9)$ & $29(11.2)$ \\
\hline \multicolumn{5}{l}{ Values are $\mathrm{n}(\%)}$. \\
L, left; R, right.
\end{tabular}

presentation to the clinic was $33.5 \%$. One hundred and forty eight of the 260 patients with dactylitis (57\%) were male and $43 \%$ were female (table 1). Their mean (SD) age was 42 (12.8) years, which was on average 8.0 (8.7) years after the onset of psoriatic arthritis.

\section{Natural history}

One hundred and thirteen of the 260 patients with dactylitis $(43 \%)$ had dactylitis in a single digit, and 147 (57\%) had multiple digits affected. The maximum number of digits affected in a single patient at any one time was 10 (present in three patients $(1 \%))$. The mean number of digits affected per patient was 2. Recurrent or persistent dactylitis occurred in the same digit in 103 of 236 patients (44\%) seen on more than one occasion.

\section{Distribution}

Information on the exact digit involved was available for 259 patients. Dactylitis was asymmetrical in 150 (58\%) of the patients. The right side was affected more often than the left (33\% v25\%). Thirty one (12\%) of the patients had dactylitis in both the hands and feet; $58(22 \%)$ had it in the hands only; $170(66 \%)$ had it only in the feet. Thus $201(78 \%)$ of the patients had dactylitis in the feet, and 89 (34\%) in the hands. The mean number of digits with dactylitis in the feet was 1.7 (range 0 to 10 ) and in the hands it was 0.6 (range 0 to 6 ). The most frequently affected digit in both hands was the second digit (the index finger). The third digit (middle finger) was the second most commonly involved. In the feet the fourth digit was the most often affected, again usually bilaterally (table 2).

\section{Radiological damage}

Pre- and post-dactylitis $x$ rays to allow comparisons were available for 185 patients. The mean (SD) length of time between the occurrence of dactylitis and the pre-dactylitis

Table 3 Radiological damage in 185 patients with dactylitis

\begin{tabular}{lcc}
\hline & No of digits/joints & $\begin{array}{l}\text { Radiological } \\
\text { damage }\end{array}$ \\
\hline Hands & 104 & $57(55 \%)$ \\
Acute dactylitis & 1746 & $577(27 \%)^{*}$ \\
No dactylitis & 104 & $34(33 \%)$ \\
MCP joints & 104 & $32(31 \%)$ \\
PIP joints & 88 & $27(31 \%)$ \\
DIP joints & & \\
Feet & 321 & $93(29 \%)$ \\
Acute dactylitis & 1529 & $306(20 \%)^{*}$ \\
No dactylitis & 321 & $82(26 \%)$ \\
MTP joints & 59 & $13(20 \%)$ \\
First IP joint & & \\
\hline *Significant difference between affected and unaffected digits \\
(p<0.0005). \\
DIP, distal interphalangeal; IP, interphalangeal; MTP, \\
metatarsophalangeal; PIP, proximal interphalangeal.
\end{tabular}


$x$ rays was 0.5 (1.3) years, and the post-dactylitis $x$ rays, 7.4 (4.5) years. In these 185 patients there were 3700 digits available for comparison. Dactylitis had occurred in 425 of these digits, 104 in the hands and 321 in the feet. Significantly more radiological damage was observed in those digits affected by dactylitis than in unaffected digits $(p<0.0003)$. Of the 104 episodes of dactylitis recorded in the hands, 57 (55\%) showed evidence of radiological progression, compared with 577 of the remaining 1746 digits (27\%) $(p<0.0001)$. Looking at the individual joints of the affected digit (metatarsophalangeal, proximal interphalangeal, distal interphalangeal), there was no significant difference in incidence of radiological progression $(32 \%, 31 \%$, and $31 \%$, respectively). Examining the feet, 93 of the 321 dactylitis episodes (29\%) had radiological evidence of damage at follow up. Radiological progression was significantly greater than the $20 \%$ seen in the unaffected digits $(p<0.0004)$. The proximal interphalangeal and distal interphalangeal joints of the lateral four toes were not included in the assessment, which may have artificially lowered the rate of damage for dactylitic digits in the feet. In the first toe, for which both metatarsophalangeal and interphalangeal joint data were available, there was no significant difference in progression (26\% and $20 \%$, respectively). Though dactylitis was less frequent in the hands $(36 \%)$ than in the feet $(77 \%)$, when it did occur, the risk of apparent radiological damage was slightly greater, even when comparing metacarpophalangeal and metatarsophalangeal joints (32\% v 26\%) (table 3). One hundred and fifty five digits had recurrence of dactylitis in the course of follow up. Digits with recurrent dactylitis more often had radiological progression that those without (69 of $155(45 \%) v 81$ of $270(30 \%) ; \mathrm{p}=0.003)$.

\section{DISCUSSION}

Dactylitis is one of the hallmark features of the spondyloarthropathies, and especially psoriatic arthritis. In the Toronto cohort of patients with psoriatic arthritis it is a common event, occurring in $48 \%$ of patients at some time. Dactylitis was most often seen at the first clinic visit (69\%), even though on average this was eight years after the onset of the disease. The high frequency at the first clinic visit may reflect the fact that dactylitis is a well recognised and distinctive sign that triggers referral to a specialist clinic.

Trauma has been described as a possible cause or trigger for psoriatic arthritis, at least on an anecdotal basis. ${ }^{12}$ The distribution of dactylitis tends to support this assumption, with a higher prevalence in the feet, right hand, and index fingers.

The natural history of psoriatic arthritis, particularly with regard to its radiological progression, is yet to be well described. We do not know if damage only occurs in clinically affected joints, or what markers predict progression for a single joint. MRI studies suggest that dactylitis is primarily an inflammation of the flexor tenosynovial sheaths, with limited joint involvement. A study using ultrasound on a limited group of patients showed evidence of joint involvement with articular synovitis in up to $52 \%$ of cases. ${ }^{78}$ Why this should be is unclear, but it may reflect imaging differences in the sensitivity of testing between the two populations. Alternatively they may have been investigated at different stages of the natural history of dactylitis. In the ultrasound cohort, ${ }^{7}$ radiographic evidence of joint erosion was already evident in 50\% of digits with dactylitis, compared with only one case in the MRI study. The level of joint involvement detected on ultrasound is, however, consistent with the proportion of patients developing radiological damage after an episode in the present study.
Our study confirms that dactylitis is not just a disease of the tenosynovium but is also associated with radiologically evident erosive damage to joints. There does not appear to be any preference for damage to a particular small joint in an affected digit, confirming that dactylitis affects the whole finger with equal severity. Although we did not assess the proximal and distal interphalangeal joints of the toes systematically (except for the interphalangeal joint of the first toe), it seems likely that they will also be adversely affected. Thus the impact of the dactylitis on radiological change may have been underestimated.

Do these findings have implications for therapy? Psoriatic arthritis can be highly destructive, as demonstrated by "arthritis mutilans." It is not known whether disease modifying anti-rheumatic drugs or local treatment with steroid injections have an affect on radiological progression. A recent study suggests that the anti-tumour necrosis factor agent etanercept may halt radiological progression in psoriatic arthritis. ${ }^{13}$ However, dactylitis was not assessed as an outcome measure in that study.

\section{Conclusions}

Dactylitis occurs commonly among patients with psoriatic arthritis. It can be destructive, and its presence should prompt the physician to start specific treatment. In clinical practice, injection of corticosteroid in the tenosynovial sheath and joints can produce some resolution of symptoms, especially when done under ultrasound guidance. ${ }^{7}$ As all joints in an affected digit are equally prone to radiological damage, a case could be made for injecting all the joints. Whether this will alter the outcome is as yet unknown.

\section{Authors' affiliations}

J E Brockbank, C T Schentag, The Psoriatic Arthritis Program, Centre for Prognosis Studies in the Rheumatic Diseases, University Health Network, Toronto Western Hospital, Toronto, Ontario, Canada

M Stein, D D Gladman, University of Toronto Rheumatic Disease Unit, The Psoriatic Arthritis Program, Centre for Prognosis Studies in The Rheumatic Diseases, Toronto

\section{REFERENCES}

1 Gladman DD, Rahman P. Psoriatic arthritis. In: Ruddy S, Harris ED, Sledge CB Budd RC, Sergent JS, eds. Kelly's textbook of rheumatology, 6th ed. Philadelphia: WB Saunders Co, 2001:1071-9.

2 Gladman DD. Psoriatic arthritis. Baillière's Clin Rheumatol 1995;9:319-29.

3 Moll JMH, Wright V. Psoriatic arthritis. Semin Arthritis Rheum 1973:3:55-78.

4 Veale $D$, Rogers S, Fitzgerald $O$. Classification of clinical subsets in psoriatic arthritis. Br J Rheumatol 1994;33:133-8.

5 Fournie B, Crognier L, Arnaud C, Zabraniecki L, Lascaux-Lefebvre V, Marc V et al. Proposed classification criteria of psoriatic arthritis. Rev Rhum Engl Ed 1999;66:446-56.

6 Rothschild BM, Pingitore C, Eaton M. Dactylitis: implications for clinical practice. Semin Arthritis Rheum 1998;28:41-7.

7 Kane D, Gearney T, Bresnihan B, Gibney R, Fitzgerald O. Ultrasonography in the diagnosis and management of psoriatic dactylitis. J Rheumatol 1999;25:1746-51

8 Olivieri I, Barozzi L, Favaro L, Pierro A, de Matteis M, Borghi C, et al. Dactylitis in patients with seronegative spondyloarthropathy. Arthritis Rheum 1996;39:1524-8.

9 Gladman DD, Shuckett R, Russell ML, Thorne JC, Schachter RK. Psoriatic arthritis - clinical and laboratory analysis of 220 patients. Q J Med 1987;62:127-41.

10 Gladman DD, Farewell V, Buskila D, Goodman R, Hamilton L, Langevitz P, et al. Reliability of measurements of active and damaged joints in psoriatic arthritis. J Rheumatol 1990;17:62-4.

11 Rahman P, Gladman DD, Cook RJ, Zhou Y, Young G, Salonen D Radiological assessment in psoriatic arthritis. Br J Rheumatol 1998;37:760-5.

12 Punzi L, Pianon M, Bertazzolo N, Faggio U, Rizzi E, Rossini P, et al. Clinical, laboratory and immunogenetic aspects of post-traumatic psoriatic arthritis: a study of 25 patients. Clin Exp Rheumatol 1998;16:277-81.

13 Ory P, Sharp JT, Salonen D, Rubenstein J, Mease PJ, Kivitz AJ, et al. Etanercept (ENBREL ${ }^{\circledR}$ ) inhibits radiographic progression in patients with psoriatic arthritis [abstract]. Arthritis Rheum 2002;46(suppl 9):S196. 